\section{Stephen J. Price, PhD, FRCS}

Neurosurgery Division, Department of Clinical Neurosciences and Wolfson Brain Imaging Centre, University of Cambridge, Cambridge Biomedical Campus, Cambridge, United Kingdom

\section{Correspondence:}

Stephen J. Price, PhD, FRCS(Neuro.Surg), Neurosurgery Division,

Department of Clinical Neurosciences, Box 167,

Cambridge Biomedical Campus,

Cambridge CB2 OQQ, UK

E-mail: sjp58@cam.ac.uk

Copyright $\odot 2015$ by the Congress of Neurological Surgeons.

\title{
Imaging Markers of Isocitrate Dehydrogenase-1 Mutations in Gliomas
}

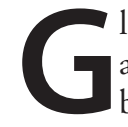

lioblastomas (GBM) are the commonest and most aggressive primary tumor of the brain. They are associated with an appalling prognosis, with survival of a matter of weeks in untreated patients, improving to 14 to 16 months with radiotherapy with concomitant and adjuvant chemotherapy. ${ }^{1,2}$ In this era of molecular biology, diagnosis still relies on the World Health Organization classification system, which only requires a tumor to have features of an astrocytic tumor with either necrosis or microvascular proliferation on light microscopy. ${ }^{3}$ Yet, it is clear that GBMs form a very heterogeneous group of tumors. Although there are recognized histological variants (eg, giant-cell GBM, GBM with oligodendroglial differentiation), it is doubtful how useful this distinction is in predicting outcome. ${ }^{4}$ It is clear that we need to be able to better subtype GBMs to consider individualizing treatment.

We have known for some time that GBMs can arise from 2 pathways: either as a primary de novo GBM or as a secondary GBM from a pre-existing low-grade glioma. These 2 subtypes have different mutations and activate different pathways within the cells. ${ }^{5}$ The discovery of mutations of the isocitrate dehydrogenase (IDH) gene, an early marker of astrocytic tumor development, ${ }^{6}$ has led to the realization that some GBMs have this mutation. ${ }^{7-9}$ This cohort of patients has a far better prognosis, with a median survival of 31 months compared with 15 months in patients with wild-type IDH. ${ }^{8}$

\section{IDH-1 MUTATIONS IN GLIOMAS}

IDH exists in 2 major forms within cells: the cytosolic IDH-1 or the mitochondrial IDH-2. The normal role is to convert isocitrate from the Krebs cycle to $\alpha$-ketoglutarate and in the process replenish $\mathrm{NADPH}$ and $\mathrm{NADH}$. $\alpha$-Ketoglutarate is also a cofactor for histone demethylase and is involved in regulation of the genome. It is thought that $\alpha$-ketoglutarate provides a mechanism for cells to deal with oxidative stresses (eg, that produced by chemotherapy or radiotherapy). Mutations of the
IDH gene result in failure to synthesize $\alpha$-ketoglutarate; instead, it leads to the production of the oncometabolite 2-hydroxyglutarate (2-HG). ${ }^{10}$ This results in the cell being unable to respond to oxidative stresses and may increase sensitivity to chemotherapy and radiotherapy. 2-HG also inhibits histone demethylases and can lead to the dysregulation of epigenetic and gene expression. In addition, 2-HG induces the hypoxia-inducible factor- $1 \alpha$ subunit, ${ }^{11}$ leading to alteration of tumor angiogenesis and growth in low oxygen concentrations. ${ }^{12}$

Mutations of the IDH gene are found in $>80 \%$ of World Health Organization grade II and III gliomas ${ }^{8}$ and in $12 \%$ of GBMs, mostly secondary GBMs. ${ }^{7,8}$ In gliomas, the majority of mutations involve IDH-1 rather than IDH-2. ${ }^{8}$ Examination of the mutation in gliomas has shown that these mutations are predominantly a point mutation of histidine replacing arginine in codon 132 (R132H mutation). ${ }^{6,7}$ This can be detectable with an immunohistochemical test ${ }^{13}$ that is able to identify most mutations very simply in paraffinembedded specimens. Recent reviews of immunohistochemical diagnosis have suggested that it can identify IDH-1 mutations with a concordance rate to sequencing of $88 \%$ to $99 \%$. $^{14}$

If IDH-1 mutations can be identified in at least $90 \%$ of cases with a simple immunohistochemical test, is there a need to develop imaging methods to do this noninvasively? There are potential advantages:

1. IDH-1 mutation may influence surgical decision making. A recent study by Beiko et $\mathrm{al}^{15}$ showed that IDH-1-mutated highgrade gliomas were more amenable to complete resection of enhancing tumor and had an improved survival with resection of the nonenhancing components.

2. Therapeutic blockade of IDH-1. There is much interest in developing selective inhibitors of the R132H IDH-1 mutation. Studies suggest that this slows tumor growth and promotes glioma differentiation in IDH-1mutated tumors but not in IDH-1 wild-type tumors. $^{16}$ 


\section{IDH-1 MUTATIONS AND CONVENTIONAL MAGNETIC RESONANCE IMAGING}

Studies comparing IDH-1 mutation status with conventional imaging have shown some differences between IDH-1-mutated and IDH-1 wild-type gliomas (Figure 1). IDH-1 tumors are more commonly found in the frontal lobe, ${ }^{15,17-22}$ whereas IDH-1 wild-type tumors commonly involve the insular region. ${ }^{23}$ IDH-1-mutated high-grade gliomas are more likely to be nonenhancing ${ }^{17,19}$ and in those exhibiting enhancement may have a distinct enhancing border ${ }^{23}$ although other studies suggest that this does not differentiate these tumors. ${ }^{18}$ In anaplastic oligodendrogliomas, wild-type tumors are more likely to exhibit a ring-like contrast enhancement pattern. $^{20}$

None of these features, however, are diagnostic. Carrillo et $\mathrm{al}^{17}$ have shown that combining the presence of enhancement, tumor size, and the presence of cysts or satellite lesions can predict IDH1 -mutated gliomas with a sensitivity of $71.4 \%$ and specificity of 99\%. This needs testing prospectively.

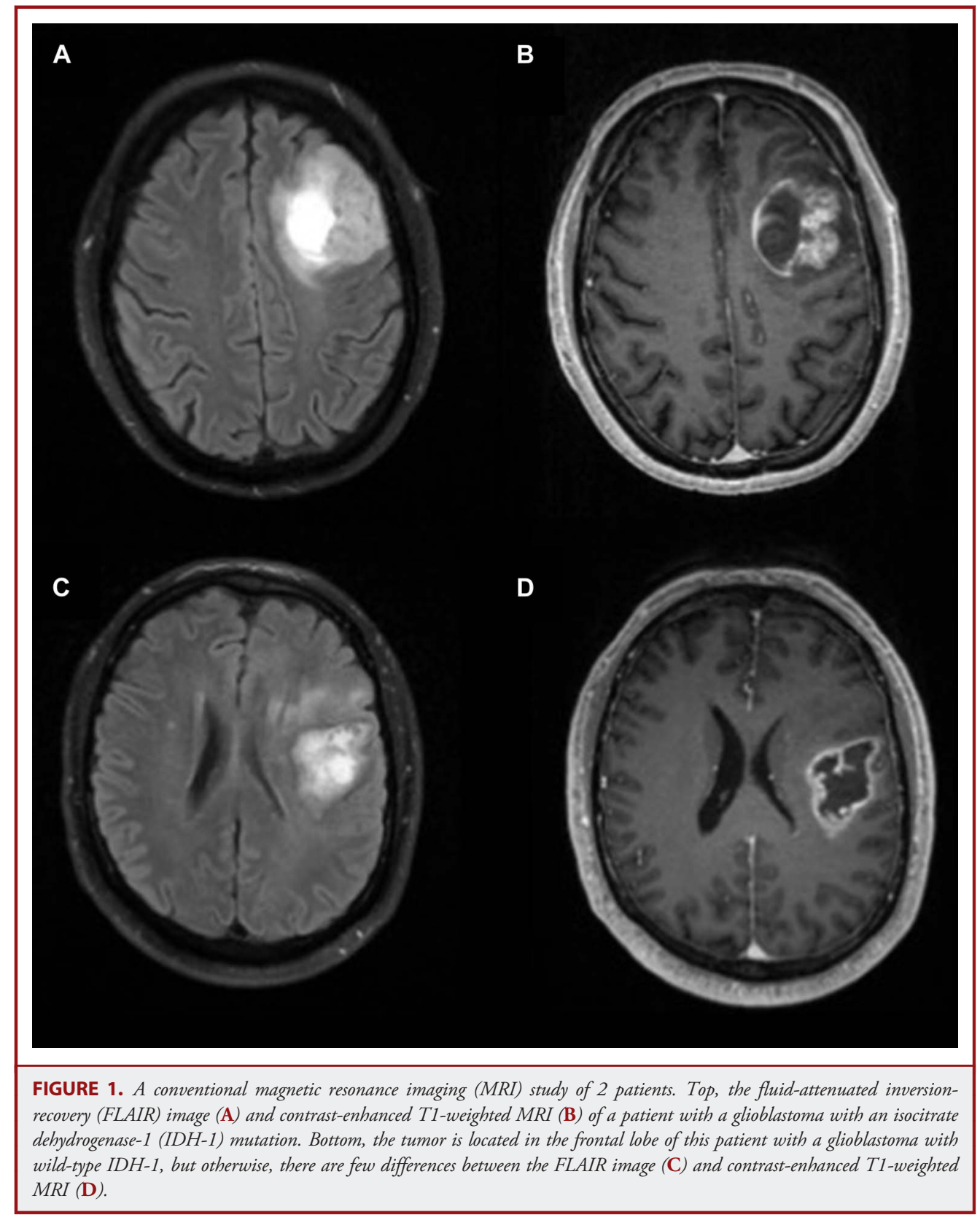




\section{MEASURING 2-HG WITH MAGNETIC RESONANCE SPECTROSCOPY}

As previously mentioned, IDH-1-mutated gliomas lead to accumulation of 2-HG within the tumor. Cell culture studies suggest a 100-fold increase in IDH-1-mutated cells to concentrations of 5 to $35 \mathrm{mmol} / \mathrm{L},{ }^{10}$ a level similar to that detected with high-resolution magic angle spectroscopy of ex vivo biopsy specimens. A number of studies have shown that 2-HG can be detected in ex vivo biopsy specimens with high-resolution magic angle spectroscopy. ${ }^{24-27}$ The concordance between detecting 2-HG and IDH-1 mutation status is $86.4 \% .{ }^{25}$ Detection of 2-HG identifies IDH-1 mutations with a sensitivity of $96 \%$ and specificity of $95.2 \%$, giving an overall accuracy of $97.8 \%{ }^{26}$ 2-HG levels within these biopsy specimens increase with increased tumor grade and increased MIB-1 proliferation rate. ${ }^{25}$ It is negatively correlated with vessel density and ADC. ${ }^{25}$ In progressive low-grade gliomas, an increase in 2-HG can identify glioma transformation to a higher grade. ${ }^{27}$
Recent studies have also shown that 2-HG can be detected in patients with gliomas using single voxel magnetic resonance spectroscopy (MRS). ${ }^{24,28,29}$ These studies suggest that it can identify IDH-1 mutations with high sensitivity. ${ }^{24,28,29}$ Largescale validation studies are required to qualify this as a biomarker of IDH-1 mutation status.

The difficulty of identifying 2-HG with spectroscopy is that the spectrum for 2-HG occurs between 2.6 and $2.4 \mathrm{ppm}$, a region shared with glutamate, glutamine, and $N$-acetyl-L-aspartate. In fact, the spectrum overlaps the glutamate and glutamine peaks (Figure 2). Interestingly, the glutamate and glutamine peaks are not affected by the IDH-1 mutation status, suggesting that the differences are due to changes in 2-HG. Attempts using standard short-echo MRS methods have shown a high sensitivity but low specificity with a false-positive rate of $26 \%{ }^{28}$ Longer-echo MRS methods that involve spectral editing and fitting the spectra to the 2-HG peak can improve the diagnostic ability ${ }^{29}$ without adding too much complexity. More accurate and sensitive methods include different MRS techniques in which overlapping

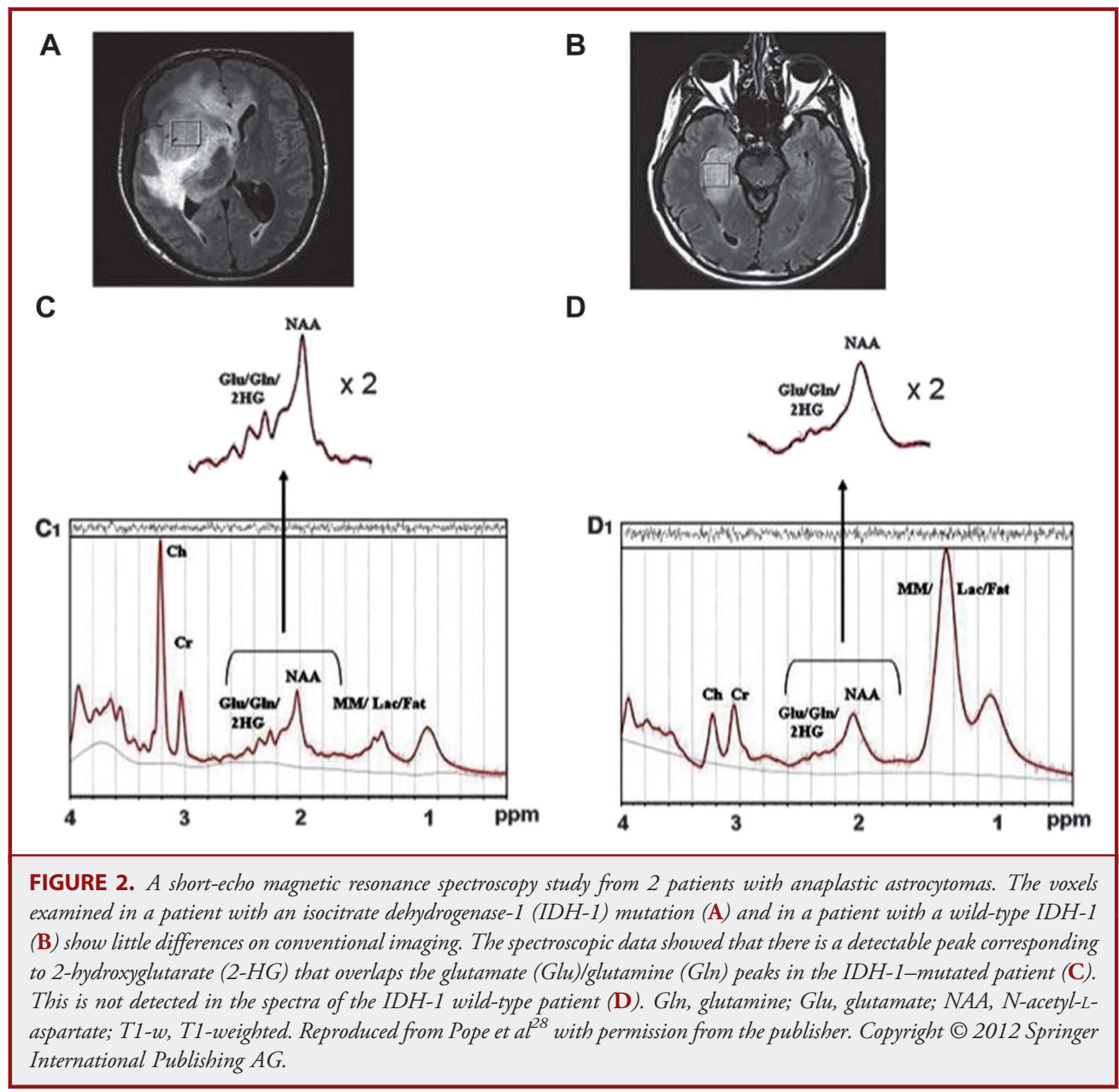


resonances are removed ${ }^{24,29}$ or a 2-dimensional correlation MRS method. ${ }^{24}$ These methods have been used by some groups, but they are not standard methods and are difficult to apply on standard clinical machines. Overall, there is yet to be a consensus on the best method of measuring 2-HG that balances accuracy and ease of use to ensure that it becomes part of routine clinical practice.

\section{IDENTIFYING CHANGES IN MICROSTRUCTURE WITH DIFFUSION AND DIFFUSION TENSOR IMAGING}

Diffusion MR provides methods that can probe the microscopic physical properties of tissues and the influence of cellularity and patterns of cellular organization. The biophysical properties of diffusion imaging are reviewed elsewhere. ${ }^{30}$

Tan et $\mathrm{al}^{31}$ investigated the role of diffusion and diffusion tensor methods to differentiate between IDH-1-mutated and IDH-1 wild-type astrogliomas. They found that grade II and III tumors have an increase in normalized apparent diffusion coefficient (ADC) and a reduction in normalized fractional anisotropy (FA) in the IDH-1-mutated tumors and postulated that this was due to differences in tumor cellularity and angiogenesis. They were able to calculate cutoff values for the ADC and FA that provided good sensitivity and specificity for determining the IDH-1 mutation status.

Diffusion tensor imaging has commonly been used to study the peritumoral region. Tan et $\mathrm{al}^{31}$ found no difference in these markers in the peritumoral regions, but previous studies have shown that FA is an insensitive marker for identifying the invasive margin. $^{32}$ Using a tensor decomposition method splits the information in the diffusion tensor into an isotropic component (which measures magnitude of diffusion) and anisotropic component (which is sensitive to directionality of diffusion). ${ }^{33,34}$ Our previous studies have shown that these methods can identify 3 invasive phenotypes that predict the pattern of tumor progression ${ }^{35}$ and time to tumor progression. ${ }^{36}$ In a cohort of GBM patients, virtually all of the IDH-1-mutated tumors as detected with $\mathrm{R} 132 \mathrm{H}$ immunohistochemistry exhibited a minimally invasive phenotype, whereas only $8 \%$ of IDH-1 wild-type tumors exhibited this phenotype 37 (Figure 3). This suggests different invasive behaviors between IDH-1-mutated and wild-type tumors. These may explain the differences in survival seen with extended resection of IDH-1-mutated tumors. ${ }^{15}$

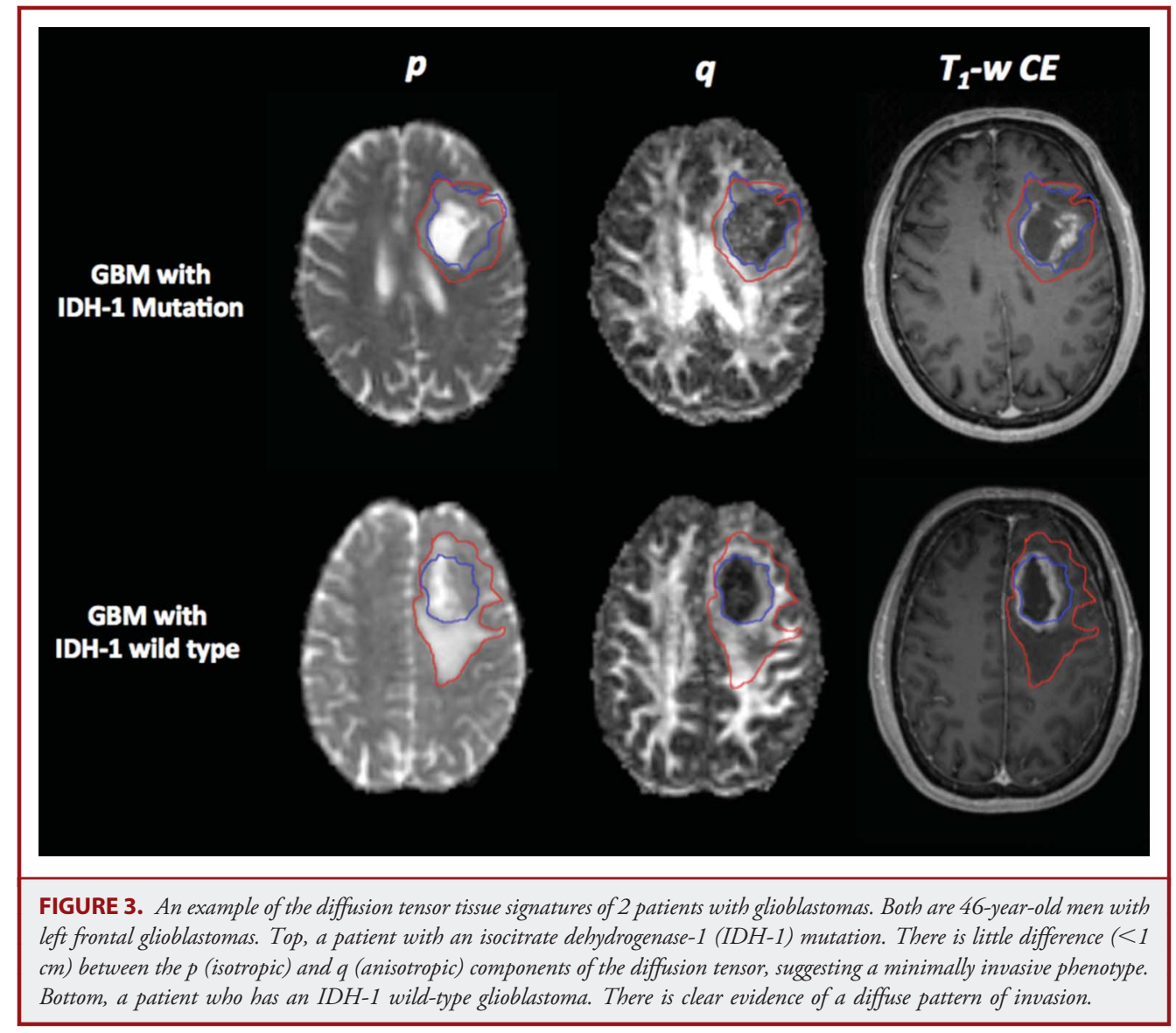




\section{CONCLUSION}

IDH-1-mutated gliomas are an important subgroup of gliomas with an improved prognosis. Imaging methods, particularly spectroscopic and diffusion, are able to identify this subgroup. Further studies are required to prospectively assess these biomarkers in a multicenter setting to allow their use in routine clinical practice.

\section{Disclosures}

Dr Price is supported by a Clinician Scientist Fellowship from the National Institute of Health Research. The author has no personal, financial, or institutional interest in any of the drugs, materials, or devices described in this article.

\section{REFERENCES}

1. Stupp R, Mason WP, van den Bent MJ, et al. Radiotherapy plus concomitant and adjuvant temozolomide for glioblastoma. $N$ Engl J Med. 2005;352(10):987-996.

2. Gilbert MR, Wang M, Aldape KD, et al. Dose-dense temozolomide for newly diagnosed glioblastoma: a randomized phase III clinical trial. J Clin Oncol. 2013;31 (32):4085-4091.

3. Louis DN, Ohgaki H, Wiestler OD, Cavenee WK. WHO Classification of Tumors of the Central Nervous System. Vol 4. Lyon: IARC; 2007.

4. Elmahdi A, Frary AJ, Scotton WJ, O’Donovan DG, Price SJ. Glioblastomas with oligodendroglial component have the same clinical phenotype as classical glioblastomas. Br J Neurosurg. 2013;27(4):419-424.

5. Ohgaki $H$, Kleihues P. Genetic alterations and signaling pathways in the evolution of gliomas. Cancer Sci. 2009;100(12):2235-2241.

6. Watanabe T, Nobusawa S, Kleihues P, Ohgaki H. IDH1 mutations are early events in the development of astrocytomas and oligodendrogliomas. Am J Pathol. 2009;174(4):1149-1153

7. Parsons DW, Jones S, Zhang X, et al. An integrated genomic analysis of human glioblastoma multiforme. Science. 2008;321(5897):1807-1812.

8. Yan H, Parsons DW, Jin G, et al. IDH1 and IDH2 mutations in gliomas. NEnglJ Med. 2009;360(8):765-773

9. Verhaak RG, Hoadley KA, Purdom E, et al. Integrated genomic analysis identifies clinically relevant subtypes of glioblastoma characterized by abnormalities in PDGFRA, IDH1, EGFR, and NF1. Cancer cell. 2010;17(1):98-110.

10. Dang L, White DW, Gross S, et al. Cancer-associated IDH1 mutations produce 2-hydroxyglutarate. Nature. 2009;462(7274):739-744.

11. Prensner JR, Chinnaiyan AM. Metabolism unhinged: IDH mutations in cancer Nat Med. 2011;17(3):291-293.

12. Zhao $\mathrm{S}$, Lin $\mathrm{Y}, \mathrm{Xu}$ W, et al. Glioma-derived mutations in IDH1 dominantly inhibit IDH1 catalytic activity and induce HIF-1alpha. Science. 2009;324(5924): 261-265.

13. Capper D, Weissert S, Balss J, et al. Characterization of R132H mutation-specific IDH1 antibody binding in brain tumors. Brain Pathol. 2010;20(1):245-254.

14. Zou Y, Bai HX, Wang Z, Yang L. Comparison of immunohistochemistry and DNA sequencing for the detection of IDH1 mutations in gliomas [published online ahead of print January 3, 2015]. Neuro Oncol. doi: 10.1093/neuonc/nou351. Available at: http:// neuro-oncology.oxfordjournals.org/content/17/3/477.long. Accessed February 14, 2015

15. Beiko J, Suki D, Hess KR, et al. IDH1 mutant malignant astrocytomas are more amenable to surgical resection and have a survival benefit associated with maximal surgical resection. Neuro Oncol. 2014;16(1):81-91.

16. Rohle D, Popovici-Muller J, Palaskas N, et al. An inhibitor of mutant IDH1 delays growth and promotes differentiation of glioma cells. Science. 2013;340(6132): 626-630.
17. Carrillo JA, Lai A, Nghiemphu PL, et al. Relationship between tumor enhancement, edema, IDH1 mutational status, MGMT promoter methylation, and survival in glioblastoma. AJNR Am J Neuroradiol. 2012;33(7):1349-1355.

18. Sonoda Y, Shibahara I, Kawaguchi T, et al. Association between molecular alterations and tumor location and MRI characteristics in anaplastic gliomas. Brain Tumor Pathol. 2015;32(2):99-104.

19. Qi S, Yu L, Li H, et al. Isocitrate dehydrogenase mutation is associated with tumor location and magnetic resonance imaging characteristics in astrocytic neoplasms. Oncol Lett. 2014;7(6):1895-1902.

20. Reyes-Botero G, Dehais C, Idbaih A, et al. Contrast enhancement in $1 \mathrm{p} / 19 \mathrm{q}-$ codeleted anaplastic oligodendrogliomas is associated with $9 \mathrm{p}$ loss, genomic instability, and angiogenic gene expression. Neuro Oncol. 2014;16(5):662-670.

21. Zhang CB, Bao ZS, Wang HJ, et al. Correlation of IDH1/2 mutation with clinicopathologic factors and prognosis in anaplastic gliomas: a report of 203 patients from China. J Cancer Res Clin Oncol. 2014;140(1):45-51.

22. Ellingson BM, Lai A, Harris RJ, et al. Probabilistic radiographic atlas of glioblastoma phenotypes. AJNR Am J Neuroradiol. 2013;34(3):533-540.

23. Metellus P, Coulibaly B, Colin C, et al. Absence of IDH mutation identifies a novel radiologic and molecular subtype of WHO grade II gliomas with dismal prognosis. Acta Neuropathol. 2010;120(6):719-729.

24. Andronesi OC, Kim GS, Gerstner E, et al. Detection of 2-hydroxyglutarate in IDH-mutated glioma patients by in vivo spectral-editing and $2 \mathrm{D}$ correlation magnetic resonance spectroscopy. Sci Transl Med. 2012;4(116):116ra4.

25. Elkhaled A, Jalbert LE, Phillips JJ, et al. Magnetic resonance of 2-hydroxyglutarate in IDH1-mutated low-grade gliomas. Sci Transl Med. 2012;4(116):116ra5.

26. Kalinina J, Carroll A, Wang L, et al. Detection of "oncometabolite" 2hydroxyglutarate by magnetic resonance analysis as a biomarker of IDH1/2 mutations in glioma. J Mol Med (Berl). 2012;90(10):1161-1171.

27. Elkhaled A, Jalbert L, Constantin A, et al. Characterization of metabolites in infiltrating gliomas using ex vivo ${ }^{1} \mathrm{H}$ high-resolution magic angle spinning spectroscopy. NMR Biomed. 2014;27(5):578-593.

28. Pope WB, Prins RM, Albert Thomas M, et al. Non-invasive detection of 2hydroxyglutarate and other metabolites in IDH1 mutant glioma patients using magnetic resonance spectroscopy. J Neurooncol. 2012;107(1):197-205.

29. Choi C, Ganji SK, DeBerardinis RJ, et al. 2-hydroxyglutarate detection by magnetic resonance spectroscopy in IDH-mutated patients with gliomas. Nat Med. 2012;18(4):624-629.

30. Price SJ, Tozer DJ, Gillard JH. Methodology of diffusion-weighted, diffusion tensor and magnetisation transfer imaging. Br J Radiol. 2011;84(Spec No 2): S121-S126.

31. Tan WL, Huang WY, Yin B, Xiong J, Wu JS, Geng DY. Can diffusion tensor imaging noninvasively detect IDH1 gene mutations in astrogliomas? A retrospective study of 112 cases. AJNR Am J Neuroradiol. 2014;35(5):920-927.

32. Price $S$, Jena $\mathrm{R}$, Burnet $\mathrm{N}$, et al. Improved delineation of glioma margins and regions of infiltration with the use of diffusion tensor imaging: an image-guided biopsy study. AJNR Am J Neuroradiol. 2006;27(9):1969-1974.

33. Price S, Peña A, Burnet N, et al. Tissue signature characterisation of diffusion tensor abnormalities in cerebral gliomas. Eur Radiol. 2004;14(10):1909-1917.

34. Peña A, Green H, Carpenter T, Price S, Pickard J, Gillard J. Enhanced visualization and quantification of magnetic resonance diffusion tensor imaging using the p:q tensor decomposition. Br J Radiol. 2006;79(938):101-109.

35. Price S, Jena R, Burnet N, Carpenter T, Pickard J, Gillard J. Predicting patterns of glioma recurrence using diffusion tensor imaging. Eur Radiol. 2007;17(7): 1675-1684.

36. Mohsen LA, Shi V, Jena R, Gillard JH, Price SJ. Diffusion tensor invasive phenotypes can predict progression-free survival in glioblastomas. $\mathrm{Br} J$ Neurosurg. 2013;27(4):436-441.

37. Price SJ, Boonzaier NR, Lupson V, Larkin T. BrainLab neurosurgery award 196: IDH-1 mutated glioblastomas have a less invasive phenotype than IDH-1 wild type glioblastomas: a diffusion tensor imaging study. Neurosurgery. 2014;61:225. 